\title{
Intérêt d'un exercice centré sur la capacité d'auto-validation des connaissances au cours d'un dispositif d'apprentissage par problèmes
}

\section{Relevance of a knowledge self-validation exercise during a problem-based learning seminar}

\author{
Anne COLLARD ${ }^{1}$, France MELOT ${ }^{2}$, Jean-Pierre BOURGUIGNON ${ }^{1,3}$ \\ ${ }^{1}$ Université de Liège, Institut de formation et recherche en enseignement supérieur, 4000 Liège, Belgique \\ ${ }^{2}$ Université de Liège, Faculté de médecine, Bureau pédagogique, 4000 Liège, Belgique \\ ${ }^{3}$ Université de Liège, Faculté de médecine, Département des sciences cliniques, 4000 Liège, Belgique
}

Manuscrit reçu le 30 août 2013 ; commentaires éditoriaux formulés aux auteurs le 26 janvier et le 31 mai 2014 ; accepté pour publication le 16 juin 2014

\begin{abstract}
Mots-clés Apprentissage par problèmes ; raisonnement biomédical ; auto-évaluation des connaissances

Résumé - Contexte : Les dispositifs pédagogiques recourant aux principes et au format de l'apprentissage par problèmes (APP) exigent que l'étudiant sélectionne les informations pertinentes d'un problème clinique et les relie par des liens explicatifs au regard de la physiopathologie sous-jacente. Dans le cadre de ces séances en petits groupes, l'interaction avec les pairs et avec l'encadrant force l'étudiant à utiliser ses connaissances antérieures, à les organiser et à les restructurer en y intégrant ses nouveaux apprentissages, en explicitant notamment les interrelations conceptuelles. À travers cet exercice, on postule que l'APP renforce le développement du raisonnement biomédical. Buts : Évaluer l'impact d'une consigne de validation des connaissances sur les éléments contenus et travaillés dans les schémas physiopathologiques au cours d'une séance retour expérimentale d'APP ; comparer avec l'évolution des schémas individuels au cours de la séance retour en l'absence de consigne particulière transmise aux étudiants. Méthodes : Un exercice d'auto-validation du champ des connaissances a été intégré à différents moments d'une séance APP. Le nombre d'items et de liens figurant sur les schémas physiopathologiques élaborés par les étudiants aux différentes étapes de la séance ont été mesurés. Les données issues d'un questionnaire sur le fonctionnement en APP et d'un débriefing ont également été recueillies. Résultats et conclusion : L'exercice de validation et de délimitation du champ des connaissances, lorsqu'il est effectué avant l'élaboration du schéma coopératif,
\end{abstract}




$\begin{array}{ll} & \begin{array}{l}\text { entraine une réduction d'environ } 60 \% \text { du nombre d'éléments manquants identifiés après le } \\ \text { schéma coopératif et renforce donc l'apprentissage individuel, en favorisant la démarche per- } \\ \text { sonnelle de l'étudiant de la prise de conscience de ses acquis et de ses limites. }\end{array} \\ \begin{array}{l}\text { Keywords } \\ \text { Problem-based } \\ \text { learning; biomedical } \\ \text { reasoning; } \\ \text { self-assessment } \\ \text { of knowledge }\end{array} & \begin{array}{l}\text { Abstract - Background: Problem-based Learning requires that each student selects the rele- } \\ \text { vant information in a clinical case scenario and connects them by causal links explaining the }\end{array} \\ \text { encourage students to organize and structure their ideas and thoughts using knowledge base } \\ \text { and explaining the relation between them. This PBL exercise helps consolidate biomedical } \\ \text { reasoning. Aim: The objective of the current study is to assess how self-validating core } \\ \text { knowledge affects learning, specifically the management of schematic biomedical reasoning. } \\ \text { Methods: A self-validation of core knowledge exercise was integrated at different moments } \\ \text { in a PBL seminar. During the sessions they examine the number of items and links in stu- } \\ \text { dents' pathophysiological schemes and collect data from a questionnaire on the PBL seminar } \\ \text { and debriefing processes. Results and conclusion: The validating and delimiting of core } \\ \text { knowledge prior to developing a final cooperative scheme lead to a 60\% reduction in missing } \\ \text { items or links identified after the cooperative scheme. Accordingly the exercise provides } \\ \text { compelling evidence that stimulating the awareness of one's knowledge and limits might } \\ \text { strengthen individual learning. }\end{array}$

\section{Introduction}

\section{Problématique}

Un des objectifs fondamentaux de la formation médicale est l'apprentissage du raisonnement clinique. Un certain nombre de dispositifs pédagogiques ont été mis en place dans le but de promouvoir le développement de cette compétence. Dans ce contexte, depuis l'année 2000, la Faculté de médecine de l'Université de Liège a mis en place des séminaires d'apprentissage par problèmes (APP) tout au long de son cursus de formation ${ }^{[1]}$. L'APP a été développé pour promouvoir un engagement actif des étudiants dans leurs apprentissages et favoriser la construction de leurs connaissances, en sollicitant systématiquement la réactivation des connaissances antérieures et en suscitant explicitement différentes stratégies d'élaboration, d'organisation et de réutilisation des connaissances chez les étudiants ${ }^{[2-5]}$. En outre, l'APP permet de créer des liens avec le contexte professionnel futur où ces connaissances devront être réactivées ${ }^{[6]}$.

L'APP cherche donc à favoriser un apprentissage en profondeur et à développer l'apprentissage auto-dirigé ${ }^{[6-7]}$. L'APP soutient le développement du raisonnement hypothético-déductif, en modélisant le processus caractérisé par la génération d'hypothèses, leur évaluation et leur révision ainsi que leur synthèse. Ce processus est notamment utilisé par les étudiants et les cliniciens lorsqu'ils sont confrontés à des cas non familiers ou atypiques, pour lesquels des processus non analytiques, tels que celui de la reconnaissance de forme (pattern recognition), n'ont pas encore été développés par l'expérience ${ }^{[8]}$. À travers ces différents mécanismes, l'APP cherche à promouvoir le développement de la capacité de raisonnement ${ }^{[9]}$.

Schwartz et al. ${ }^{[10]}$ précisent que les étudiants ayant bénéficié d'un curriculum recourant à l'APP ont démontré de meilleures performances en raisonnement ainsi qu'en connaissances médicales et factuelles. Friedman et al. ${ }^{[11]}$ ont également montré que l'APP permet aux étudiants d'atteindre des habiletés interpersonnelles supérieures, une plus grande pratique de l'apprentissage continué et un plus haut niveau de satisfaction professionnelle. Plus récemment, Haak et al. ${ }^{[12]}$ ont montré que l'APP appliqué en biologie facilitait l'apprentissage et diminuait la disparité entre étudiants avec des connaissances initiales inégales. Dans le cadre de ces séances d'APP 
en petits groupes, l'interaction avec les pairs et l'encadrant permet un élargissement de la base de connaissances et le développement d'habiletés d'auto-gestion. L'encadrant et les pairs aident l'étudiant à explorer les phénomènes identifiées dans le problème et le poussent à verbaliser ses pensées. Cela force l'étudiant à organiser ses pensées et idées, et à expliciter à la fois les liens cognitifs qu'il mobilise et ceux qu'il établit, utilisant et remaniant ainsi sa base de connaissances ${ }^{[13]}$.

Une recherche antérieure a montré l'accroissement de la capacité de raisonnement et son lien avec les connaissances factuelles dès les années précliniques au cours du curriculum impliquant l'APP à la Faculté de médecine de l'Université de Liège ${ }^{[14]}$. En outre, la capacité individuelle de raisonnement s'est avérée liée à la capacité de délimiter le champ de ses connaissances, à tous les stades étudiés de la formation. Ce constat est important dans la mesure où parfois, certains étudiants privilégient le raisonnement en termes de stratégies générales, en négligeant la construction du bagage cognitif spécifique, c'est-àdire des connaissances qui sous-tendent ce raisonnement. Ceci souligne l'importance, pour un étudiant, d'être capable de délimiter ses connaissances pour valider son raisonnement ${ }^{[15-16]}$.

Le processus de raisonnement dépend de la base de connaissances, de la capacité d'analyser et de synthétiser des données ${ }^{[17-19]}$, ainsi que de la conscience et de la capacité de contrôle de ce processus de raisonnement ${ }^{[20-21]}$. Ces facteurs influencent tous les aspects du raisonnement biomédical et clinique, et peuvent eux-mêmes être améliorés lorsque l'étudiant ou le clinicien réfléchit consciemment aux informations récoltées (accréditant ou discréditant ses hypothèses), sur lesquelles ses demandes de renseignements et ses décisions cliniques sont basées. Afin de faciliter le processus de raisonnement des étudiants, il est donc important de les rendre conscients des différents facteurs qu'il implique. Cela suppose que les étudiants puissent d'une part, disposer des connaissances nécessaires et, d'autre part, les sélectionner de façon pertinente pour appréhender les informations relatives à un cas clinique donné. En effet, lorsque le praticien est confronté à un nouveau cas clinique, les éléments de ce cas vont activer des informations en mémoire. Sur la base de ses connaissances et du contexte du cas clinique, il va pouvoir appréhender le cas en sélectionnant les informations qui lui apparaissent les plus utiles. L'abstraction -ou décontextualisation- de ces éléments permet d'identifier et d'activer rapidement le schème à appliquer à cette situation. Lorsqu'il s'agit d'un nouveau cas, qui comporte des éléments que le praticien n'a jamais rencontré préalablement, le schème qui comprend le plus d'éléments concordants sera activé et identifié, puis adapté à la nouvelle situation rencontrée. Plus cette base de connaissances est structurée, plus il sera facile d'identifier et d'activer le bon schème, et d'y intégrer les nouvelles connaissances et de nouveaux liens. Identifier les connaissances maîtrisées et celles qui ne le sont pas apparait donc essentiel pour progresser de façon efficace dans le processus de raisonnement. $\mathrm{La}$ capacité de délimiter son champ de connaissance peut ainsi être considérée comme étant une part importante du processus de validation du raisonnement. Mamede et al. ${ }^{[21]}$ précisent que la qualité du diagnostic dépend de la manière dont le clinicien gère la coordination et l'intégration de ces différentes composantes, en utilisant la métacognition. La métacognition a aussi été identifiée comme caractérisant l'expertise en raisonnement ${ }^{[15]}$. Alors que le contrôle et la réflexion sur le processus de raisonnement dans son ensemble ont été abordés dans ces études, relativement peu d'études s'intéressent à cette capacité en lien avec les connaissances utilisées pour raisonner. Or, un bon raisonnement, s'il est basé sur des connaissances erronées, ne peut conduire qu'à de mauvais résultats. La validation des connaissances semble ainsi essentielle à la validation du raisonnement ${ }^{[22]}$. Par ailleurs, dans le cadre d'un apprentissage auto-dirigé tel que le propose l'APP, qui implique que l'étudiant détermine de façon autonome les connaissances à acquérir à partir d'une situation qui lui est présentée, la capacité à discriminer ce qu'il connait et ce qu'il ne connait pas s'avère indispensable ${ }^{[16]}$. 
Tableau I. Organisation du cursus médical à l'Université de Liège.

\begin{tabular}{|c|c|c|c|c|c|c|c|}
\hline \begin{tabular}{|l|} 
Années \\
du cursus
\end{tabular} & Deuxième & \multicolumn{2}{|c|}{ Troisième } & \multicolumn{2}{|c|}{ Quatrième } & \multicolumn{2}{|c|}{ Cinquième } \\
\hline $\begin{array}{l}\text { Objectifs } \\
\text { de } \\
\text { formation }\end{array}$ & \multicolumn{3}{|c|}{$\begin{array}{l}\text { Sciences de base, structure et fonctions } \\
\text { normales et pathologiques des systèmes } \\
\text { anatomiques }\end{array}$} & \multicolumn{4}{|c|}{ Sciences cliniques, diagnostic des maladies et prise en charge } \\
\hline Modules & \begin{tabular}{|l} 
Cardiaque \\
Respiratoire \\
Rénal \\
Hématologie
\end{tabular} & $\begin{array}{l}\text { Digestif } \\
\text { Endocrino- } \\
\text { métabolisme } \\
\text { Génital }\end{array}$ & \begin{tabular}{|l|} 
Nerveux \\
Locomoteur \\
Immunitaire \\
Homéostasie
\end{tabular} & \begin{tabular}{|l|} 
Cardiologie \\
Pneumologie \\
Médecine \\
Générale
\end{tabular} & \begin{tabular}{|l} 
Obstétrique et \\
néonatologie \\
Hémato- \\
oncologie \\
Immuno- \\
rhumatologie \\
Endocrinologie \\
Néphrologie \\
Dermatologie
\end{tabular} & \begin{tabular}{|l|} 
Gastroentérologie \\
Urologie \\
Obstétrique \& \\
néonatologie
\end{tabular} & $\begin{array}{l}\text { Neurologie } \\
\text { Orthopédie } \\
\text { Ophtalmologie } \\
\text { Médecine } \\
\text { Générale }\end{array}$ \\
\hline
\end{tabular}

\section{Objectifs}

La présente étude cherche à documenter certains effets d'un séminaire recourant aux principes et au format de l'APP, au cours duquel est mise en œuvre une démarche explicite d'auto-validation des connaissances, afin de renforcer la conscience que les étudiants peuvent avoir de leur processus d'apprentissage aux différentes étapes de l'analyse d'un problème clinique. En effet, prendre conscience de son processus de raisonnement peut contribuer à améliorer l'organisation de ses propres connaissances $^{[20-21]}$. À travers la pratique et le feedback, l'objectif est de faciliter le processus de métacognition qui semble être un élément central dans la capacité de raisonnement clinique ${ }^{[13]}$. Plus spécifiquement, les objectifs de cette étude sont : a) dans un premier temps, d'évaluer l'impact d'une consigne de validation des connaissances sur l'élaboration des schémas physiopathologiques dans le cadre d'une séance « retour» expérimentale d'APP; b) dans un second temps, de comparer l'évolution des schémas individuels au cours de la séance « retour» en l'absence de consigne particulière transmise aux étudiants. Ainsi, cette étude s'interroge sur la façon dont un travail de l'étudiant portant sur sa capacité de délimiter le champ de ses connaissances lui permet de valider son raisonnement et contribue ainsi à la qualité de ses apprentissages.

\section{Méthodes}

\section{Les dispositifs pédagogiques}

La procédure habituelle des séminaires d'APP en troisième année des études médicales à la Faculté de médecine de l'Université de Liège se déroule en trois temps : 1) une phase «aller», au cours de laquelle les étudiants, encadrés par un tuteur, prennent connaissance de la vignette clinique, mettent en évidence les phénomènes à expliquer, formulent des hypothèses d'explication et identifient des objectifs d'apprentissage ; 2) une phase de travail personnel de trois à cinq jours, au cours de laquelle les étudiants s'efforcent de répondre aux objectifs d'apprentissage par leur recherche dans les ouvrages de référence et réalisent à titre individuel un schéma physiopathologique (décrit plus loin) ; 3) une phase « retour», durant laquelle les étudiants construisent un schéma coopératif au tableau, sur la base de leur travail personnel. Le plus souvent, il est de la responsabilité de l'étudiant d'intégrer son schéma individuel avec le schéma collaboratif. Parfois, le tuteur passe en revue certains schémas individuels et les commente.

\section{APP expérimental (cohorte 1 : groupe d'intervention)}

Dès la deuxième année du curriculum, les enseignements sont organisés en modules autour des grands systèmes anatomiques (Tableau I). Le module 
« endocrinologie» se déroule au premier quadrimestre de la troisième année du cursus. L'APP expérimental s'est déroulé en mars 2009, après l'évaluation certificative sur la matière d'endocrinologie concernée par l'APP expérimental.

Une vignette clinique portant sur les objectifs de ce module a été rédigée. De manière à éviter toute discrimination négative vis-à-vis des étudiants non participants, la vignette portait sur les objectifs d'apprentissage déjà envisagés lors des séminaires d'APP suivis par les étudiants mais en mêlant ceux relatifs à différentes vignettes et en les proposant dans un contexte inédit.

\section{APP régulier (cohorte 2 : groupe comparatif)}

La vignette créée dans le cadre de l'APP expérimental a été utilisée dans le cadre de l'APP régulier, dans le module de formation à l'endocrinologie des étudiants de troisième année durant l'année académique suivante. Les éléments motivationnels éventuellement liés à l'APP expérimental sont donc absents, de même qu'aucune consigne d'auto-validation n'est donnée aux étudiants.

\section{Participants}

\section{APP expérimental (cohorte 1 : groupe d'intervention)}

Vingt étudiants de troisième année de baccalauréat (dénommé licence dans certains pays) ont participé volontairement à un séminaire d'APP expérimental dans le cadre de la recherche. Sur la base de leur score global aux examens de troisième année, les étudiants de cet échantillon (moyenne : 13,4/20 ; écart-type : 2,1) n'étaient pas significativement différents des autres étudiants de la même cohorte (moyenne : 13/ 20 ; écart-type : 2,3 ).

Nous avons formé trois groupes de six ou sept étudiants, encadrés chacun par un tuteur enseignant (Tableau II).

\section{APP régulier (cohorte 2 : groupe comparatif)}

Dans un second temps, les schémas physiopathologiques élaborés par ces 20 étudiants ont été comparés à ceux réalisés par un échantillon de 23 étudiants choisis de façon aléatoire en troisième année dans la cohorte suivante, répartis dans trois groupes encadrés par un tuteur. Dans ce deuxième cas, le séminaire APP n'est plus expérimental mais fait partie du programme de formation, sur base de la même vignette clinique.

\section{Recueil des données}

\section{APP expérimental (cohorte 1 : groupe d'intervention)}

\section{Schémas physiopathologiques}

Les schémas physiopathologiques créés par les étudiants lors des séances APP ont été utilisés comme mesure de l'évolution du processus de raisonnement en cours d'APP.

Les schémas physiopathologiques sont construits individuellement par chaque étudiant. Ils s'inscrivent dans le cadre général de la cartographie cognitive, bien qu'un degré de formalisme strict de la représentation produite ne soit pas en l'occurrence exigé. La consigne prescrit aux étudiants d'identifier les éléments pertinents du cas présenté et de les mettre en relation les uns avec les autres (essentiellement des relations de causalité) afin de présenter une représentation globale de la situation et de rendre compte d'une compréhension des phénomènes et mécanismes physiopathologiques impliqués dans ce problème. Ce schéma traduit la capacité d'analyse du problème de la part de l'étudiant, en l'occurrence sa capacité à sélectionner et transformer les éléments factuels dans une construction schématique et sémantique, qui permet d'évaluer la progression vers la structuration, l'analyse et la compréhension du problème. Cette capacité est essentielle au raisonnement clinique $^{[23-24]}$.

Certains étudiants construisent des schémas élaborés mettant en relation de nombreux éléments, sans pour autant les maîtriser tous et sans qu'ils n'identifient ceux qu'ils maîtrisent respectivement mieux ou moins bien. D'autres présentent des schémas simplifiés et lacunaires, comptant sur le travail coopératif pour disposer d'un schéma collectif final comme 
Tableau II. Dispositif d'apprentissage par problèmes expérimental.

\begin{tabular}{|c|c|c|c|}
\hline Groupes & $\begin{array}{c}\mathbf{A} \\
(n=7)\end{array}$ & $\begin{array}{c}\text { B } \\
(n=7)\end{array}$ & $\begin{array}{c}\mathrm{C} \\
(n=6)\end{array}$ \\
\hline \multicolumn{4}{|l|}{ Phase « aller» } \\
\hline Information sur les consignes de délimitation des connaissances & & & $\mathbf{x}$ \\
\hline \multicolumn{4}{|l|}{ Phase de travail personnel + schéma personnel } \\
\hline \multicolumn{4}{|l|}{$\begin{array}{l}\text { Phase « retour » } \\
\text { - Récolte du schéma personnel }\end{array}$} \\
\hline $\begin{array}{l}\text { - Annotation du schéma individuel (orange }=\text { douteux, vert }=\text { manque) }+ \\
\text { récolte du schéma }\end{array}$ & & $\mathbf{x}$ & $\mathbf{x}$ \\
\hline \multicolumn{4}{|l|}{$\begin{array}{l}\text { - Construction du schéma coopératif (+ schéma individuel éventuellement } \\
\text { annoté) + récolte des schémas }\end{array}$} \\
\hline $\begin{array}{l}\text { - Annotation du schéma individuel }(\text { orange }=\text { douteux, vert = manque, } \\
\text { rouge }=\text { incorrect) }+ \text { récolte du schéma }\end{array}$ & $\mathbf{x}$ & $\mathbf{x}$ & $\mathbf{x}$ \\
\hline Questionnaire & $\mathbf{x}$ & $\mathbf{x}$ & $\mathbf{x}$ \\
\hline Débriefing enregistré & $\mathbf{x}$ & $\mathbf{x}$ & $\mathbf{x}$ \\
\hline
\end{tabular}

point de référence. L'exercice proposé dans le cadre de cette recherche vise donc à impliquer les étudiants dans un travail individuel de validation ou invalidation/correction (avec justifications) des éléments de leur propre schéma physiopathologique et, par là même, de délimitation des champs du connu et de l'ignoré.

Un travail de réflexion sur les connaissances sous-jacentes à leur schéma physiopathologique a été proposé, soit uniquement en fin de séminaire d'APP, soit en début et en fin de séance « retour » d'APP. Le groupe A a suivi la procédure habituelle des séminaires d'APP décrite précédemment. Comme d'habitude, au début de la séance « retour », avant la mise en commun dans un schéma construit en coopération, le tuteur a récolté une photocopie de chaque schéma individuel élaboré durant la phase de travail personnel, identifié avec un code pour chaque étudiant. Durant la mise en commun, chaque étudiant a annoté et amendé ou non son propre schéma selon sa stratégie habituelle, aucune instruction n'étant donnée par le tuteur. À la fin du séminaire, le tuteur a récolté les schémas individuels éventuellement annotés, ainsi que la copie du schéma coopératif transcrite par chaque étudiant. Ces documents ont été photocopiés. Finalement, un exercice de délimitation de leurs connaissances sur base de leur schéma physiopatho- logique personnel leur a été demandé. L'instruction était de souligner ou d'entourer dans leur schéma (selon trois couleurs différentes) les composants (éléments mentionnés et flèches de mise en relation) qu'ils avaient identifiés comme douteux (1), ceux qu'ils avaient invalidés car incorrects (2) et ceux qu'ils avaient ajoutés car manquants (3), sachant que les éléments non marqués étaient de facto considérés comme validés - corrects. Le groupe $\mathrm{B}$ a suivi la procédure habituelle jusqu'au début de la phase « retour». À ce moment, avant la construction d'un schéma physiopathologique coopératif, les étudiants ont été invités à marquer, sur une photocopie de leur schéma initial, les composants dont ils doutaient (1) et les parties de leur schéma où ils pensaient que des informations pouvaient manquer (3). Les éléments non marqués étant considérés comme « certains ». Ces photocopies étaient alors récoltées. Ensuite, le schéma coopératif était construit, les étudiants ayant été au préalable invités, durant cette étape, à marquer dans leur schéma initial (selon trois couleurs différentes) les composants (éléments mentionnés et flèches de mise en relation) qu'ils identifiaient comme douteux et qui s'avéraient corrects (1), ceux qu'ils invalidaient car incorrects (2) et ceux qu'ils ajoutaient car manquants (3), sachant que les éléments non marqués étaient de facto validés 
Tableau III. Moyennes (Ecart-type) du nombre d'items et de liens figurés dans les schémas aux différentes étapes de la séance « retour » et du nombre d'items et de liens caractérisés lors des exercices de marquages 1 et 2.

\begin{tabular}{|c|c|c|c|c|c|c|c|c|c|c|}
\hline \multicolumn{9}{|c|}{ Evolution des schémas individuels au cours de la séance retour } & \multirow{2}{*}{\multicolumn{2}{|c|}{$\begin{array}{c}\text { Schéma coopératif } \\
\text { collectif } \\
\text { au tableau }\end{array}$}} \\
\hline & \multicolumn{2}{|c|}{$\begin{array}{l}\text { Schémas individuels } \\
\text { en début séance retour }\end{array}$} & \multicolumn{2}{|c|}{$\begin{array}{l}\text { Schémas } \\
\text { individuels après } \\
\text { l'exercice } \\
\text { de marquage } 1\end{array}$} & \multicolumn{2}{|c|}{$\begin{array}{l}\text { Schémas individuels } \\
\text { après la construction } \\
\text { du schémas } \\
\text { coopératif }\end{array}$} & \multicolumn{2}{|c|}{$\begin{array}{l}\text { Schémas } \\
\text { individuels après } \\
\text { l'exercice } \\
\text { de marquage } 2\end{array}$} & & \\
\hline Groupes & $\begin{array}{l}\text { Nbre } \\
\text { d'items }\end{array}$ & $\begin{array}{l}\text { Nbre } \\
\text { de liens }\end{array}$ & $\begin{array}{l}\text { Nbre } \\
\text { d'items }\end{array}$ & $\begin{array}{l}\text { Nbre de } \\
\text { liens }\end{array}$ & $\begin{array}{l}\text { Nbre } \\
\text { d'items } \Delta\end{array}$ & $\begin{array}{l}\text { Nbre } \\
\text { de liens } \Delta\end{array}$ & $\begin{array}{l}\text { Nbre } \\
\text { d'items }\end{array}$ & $\begin{array}{l}\text { Nbre de } \\
\text { liens }\end{array}$ & $\begin{array}{l}\text { Nbre } \\
\text { d'items }\end{array}$ & $\begin{array}{l}\text { Nbre } \\
\text { de liens }\end{array}$ \\
\hline $\mathrm{A}(N=7)^{*}$ & $42,7(9,5)$ & $48,1(13,2)$ & & & $56,3(8,2)$ & $65,3(13,5)$ & $57,6(7,5)$ & $67(12,3)$ & 56 & 66 \\
\hline $\mathrm{B}(N=7)^{*}$ & $39,4(4,3)$ & $43,3(5,2)$ & $40,1(3,7)$ & $44,7(4,5)$ & $44,6(4,1)$ & $49,6(4,1)$ & $45,6(4,1)$ & $51,4(5)$ & 38 & 49 \\
\hline $\mathrm{C}(N=6)^{*}$ & $44,2(7,4)$ & $50,8(8,9)$ & $44,2(7,4)$ & $50,8(8,9)$ & $50,7(8,7)$ & $59,3(10,2)$ & $51,3(8,3)$ & $60,8(8,7)$ & 39 & 50 \\
\hline \multicolumn{11}{|c|}{ Exercices de marquage des schémas } \\
\hline & \multicolumn{4}{|c|}{ Exercice de marquage 1} & \multicolumn{6}{|c|}{ Exercice de marquage 2} \\
\hline & \multicolumn{2}{|c|}{ Éléments douteux } & \multicolumn{2}{|l|}{$\begin{array}{l}\text { Éléments } \\
\text { manquants }\end{array}$} & \multicolumn{2}{|c|}{ Éléments douteux } & \multicolumn{2}{|c|}{ Éléments incorrects } & \multicolumn{2}{|c|}{$\begin{array}{l}\text { Éléments } \\
\text { manquants }\end{array}$} \\
\hline Groupes & $\begin{array}{l}\text { Nbre } \\
\text { d'items } \ddagger\end{array}$ & $\begin{array}{l}\text { Nbre } \\
\text { de liens }\end{array}$ & $\begin{array}{l}\text { Nbre } \\
\text { d'items }\end{array}$ & $\begin{array}{l}\text { Nbre } \\
\text { de liens }\end{array}$ & $\begin{array}{l}\text { Nbre } \\
\text { d'items }\end{array}$ & $\begin{array}{l}\text { Nbre } \\
\text { de liens }\end{array}$ & $\begin{array}{l}\text { Nbre } \\
\text { d'items }\end{array}$ & $\begin{array}{l}\text { Nbre de } \\
\text { liens }\end{array}$ & $\begin{array}{l}\text { Nbre } \\
\text { d'items } \dagger\end{array}$ & $\begin{array}{l}\text { Nbre } \\
\text { de liens } \dagger\end{array}$ \\
\hline $\mathrm{A}(N=7)$ & & & & & $3(2,2)$ & $2,5(1)$ & & $1(0)$ & $14,6(6,3)$ & $13,9(5,5)$ \\
\hline $\mathrm{B}(N=7)$ & $2,7(1,7)$ & $3,3(1,9)$ & $5,2(2,4)$ & $2,5(1,4)$ & & & $1(0)$ & $1(0)$ & $5,1(1,8)$ & $5,3(2,7)$ \\
\hline $\mathrm{C}(N=6)$ & $4,5(1,4)$ & $3,3(1)$ & $4,7(2,2)$ & $3(1)$ & & & $2(1,4)$ & $1,3(0,6)$ & $5,3(4,7)$ & $6,7(4,9)$ \\
\hline
\end{tabular}

$\Delta$ différence significative entre le groupe A et le groupe B.

$\ddagger$ différence significative entre le groupe B et $\mathrm{C}$.

$\dagger$ différence significative entre le groupe A et les autres groupes.

* différence significative entre les schémas individuels en début de séance retour et en fin de séance retour.

comme corrects. Tous les schémas individuels annotés ont alors été récoltés ainsi que le schéma éventuellement transcrit à partir du travail collaboratif. Le groupe $\mathrm{C}$ a suivi la même procédure que le groupe $\mathrm{B}$ mais ses membres ont été avertis à la fin de la séance «aller» qu'ils auraient à réaliser un exercice de délimitation de leur champ de connaissance basé sur leur schéma en début de phase « retour».

L'évolution en termes de nombre d'items et de liens, ainsi que le niveau de certitude et de compréhension de ces items et liens, ont été mesurés.

\section{Questionnaire et débriefing}

A la fin de la séance «retour», chacun des trois groupes a rempli un questionnaire (Tableau III), spécifiquement élaboré dans le cadre de l'étude, les interrogeant sur leur fonctionnement, respectivement, dans le cadre des APP réguliers et lors de cet APP expérimental. Ce questionnaire n'a pas été administré préalablement à l'APP expérimental de manière à ne pas biaiser celui-ci par le contenu du questionnaire, qui aurait indiqué aux étudiants les aspects envisagés par le travail. Les réponses des étudiants ayant participé à cet APP expérimental ont été comparées aux réponses de 43 autres étudiants de la même cohorte.

Un débriefing interrogeant les étudiants sur le déroulement de cet APP expérimental a également été réalisé et enregistré.

\section{APP régulier (cohorte 2 : groupe comparatif)}

Les étudiants ont vécu une séance d'APP traditionnelle comme décrit plus haut. Les schémas physiopathologiques remis aux tuteurs en début de séance ont été récoltés et photocopiés. Les schémas individuels ont été également récoltés en fin de séance « retour », afin de comparer si le nombre d'items et de liens présents dans ces schémas augmentait 
de la même façon après le schéma coopératif, lorsqu'aucune consigne particulière n'était donnée. Les schémas physiopathologiques d'un échantillon de ces étudiants ont été comparés à ceux des étudiants ayant participé à l'APP expérimental.

\section{Analyses statistiques}

\section{APP expérimental (cohorte 1 : groupe d'intervention)}

Étant donné le faible échantillon, nous avons réalisé des analyses statistiques non paramétriques.

\section{Pour les schémas}

Les moyennes $( \pm \mathrm{ET})$ du nombre d'items et de liens contenus dans les schémas individuels des trois groupes ont été calculées. Les différences entre les groupes en termes d'items et de liens ont été évaluées par une analyse de variance à l'aide du test de Kruskal Wallis. L'évolution des schémas en termes d'items et de liens au cours de la séance retour pour chacun des groupes a été évaluée par une analyse de variance à l'aide du test de Friedmann. Le seuil de significativité a été fixé à $p \leq 0,05$.

\section{Pour le questionnaire}

Les pourcentages des réponses choisies aux différentes questions du questionnaire ont été calculées. Les différences entre les groupes ont été évaluées par une analyse de variance à l'aide du test de Kruskal Wallis. Les différences de fonctionnement à l'APP expérimental et lors des APP intégrés au cursus de formation ont été évaluées par une analyse de variance à l'aide du test de Friedman. Le seuil de significativité a été fixé à $p \leq 0,05$.

\section{APP régulier (cohorte 2 : groupe comparatif)}

La différence entre les deux cohortes en termes de nombre d'items et de liens présents sur les schémas en début de séance "retour » a été évaluée par une analyse de variance à un facteur. L'évolution des schémas entre le début et la fin de la séance « retour »

en termes de nombre d'items et de liens selon les cohortes a été évaluée par une Anova en mesures répétées. Le seuil de significativité a été fixé à $p \leq 0.05$.

\section{Résultats}

Impact d'une consigne d'auto-validation des connaissances sur les éléments contenus et travaillés dans les schémas physiopathologiques au cours d'une séance retour expérimentale d'APP

\section{Analyse des schémas}

Le nombre d'items et de liens présents sur les schémas physiopathologiques individuels aux différentes étapes de la séance retour est significativement plus important pour les étudiants du groupe A que pour ceux du groupe $B$ après la construction du schéma coopératif $(p=0,03)$. Toutefois, une telle différence n'est pas observée avec le groupe $\mathrm{C}$, ce qui renvoie à un impact possible des différences de groupe d'étudiant et/ou de tuteurs. Ceci est renforcé par le maintien d'une différence dans le même sens au niveau du schéma coopératif, même si celle-ci n'est plus significative.

Le tableau III montre que les étudiants du groupe $\mathrm{C}$, le seul informé de la démarche de validation avant la séance retour, ont marqué un nombre significativement plus grand d'items pour lesquels ils avaient un doute ou une hésitation par rapport au groupe $\mathrm{B}(p=0,047)$. Aucune différence n'est observée pour le nombre de liens douteux, ni sur les items et liens insuffisamment détaillés ou développés.

Les étudiants du groupe A identifient significativement plus d'items $(p=0,005)$ et liens $(p=0,01)$ manquants ou incomplets par rapport aux étudiants du groupes B et C. Aucune différence n'est observée pour les items et liens douteux ou incorrects.

Une augmentation significative du nombre d'items et de liens présents dans les schémas individuels entre le début et la fin de la séance retour est observée dans les trois groupes (A : $p<0,01 ; \mathrm{B}: p<$ $0,001 ; \mathrm{C}: p<0,01)$. 


\section{Résultats du questionnaire}

Les résultats du tableau IV illustrent le fait que, dans le cadre de l'APP expérimental, les trois groupes semblent avoir travaillé de manière équivalente lors de la phase de travail personnel. Les quelques différences observées lors de la séance retour (items 32, 34 et 36) semblent plutôt dues à une différence de fonctionnement et de dynamique des différents groupes et tuteurs. Toutefois, le groupe $\mathrm{C}$, averti de la procédure avant la phase de travail personnel, donne des réponses compatibles avec un moindre investissement lors de la phase retour, ce qui peut suggérer que le travail de validation a été en partie anticipé durant la phase de travail personnel.

Le tableau V montre que, dans le cadre de l'APP régulier, de manière générale, les étudiants ayant participé à l'APP expérimental cernent davantage l'importance de distinguer les éléments qu'ils connaissent de ceux qu'ils ne connaissent pas (items 2 et 6). En ce qui concerne la construction de leur schéma lors de la phase de travail personnel, les étudiants participants déclarent mieux identifier les éléments connus, douteux et insuffisamment détaillés par rapport aux non participants (items 7, 8, 9, 13 et 15). Pour la séance retour, les participants confirment les éléments connus et complètent les éléments manquants davantage que les non participants (items 17 et 19). Ils corrigent également plus vite leur schéma après la séance retour que les non participants (items 26 et 27).

Le tableau VI présente les différences entre l'APP expérimental et l'APP régulier. Il semble que la plupart des étudiants participants cernent déjà de manière implicite les éléments qu'ils connaissent, ceux dont ils doutent et les éléments incomplets. Néanmoins, les exercices proposés sont associés à une meilleure prise de conscience de ces différents éléments (items 17-32 ; 18-33 ; 19-34 ; 21-36 ; 2237). La façon dont la mise en commun coopérative a été menée semble avoir eu un impact positif sur leur fonctionnement ; néanmoins, aucun effet significatif de cette mise en commun sur la validation du raisonnement n'a pu être démontré.

\section{Les résultats du débriefing}

De manière générale, les étudiants apprécient le coté intégratif de l'APP sur une matière déjà étudiée. Ils considèrent le travail comme rentable, permettant une bonne intégration parce que la matière a déjà été abordée. Ils ont également apprécié l'implication des tuteurs dans la mise en commun coopérative qui a permis une analyse en profondeur de la situation clinique. En ce qui concerne les exercices de délimitation des connaissances, il semblerait que les étudiants les pratiquent déjà habituellement. Néanmoins, la nécessité de faire ce travail de façon explicite a permis à certains de mieux se rendre compte de leur évolution. Les étudiants semblent cerner l'importance de distinguer ce que l'on connait de ce que l'on ne connait pas et de la capacité de s'auto-évaluer correctement. Certains parlent de ce processus d'autoévaluation comme un raisonnement en soi.

\section{Évolution des schémas individuels au cours de la séance retour en conditions non expérimentales, sans consigne particulière donnée aux étudiants}

La figure 1 illustre l'évolution du nombre d'items et de liens présents sur les schémas physiopathologiques entre le début et la fin de la séance « retour » pour le groupe d'étudiants ayant respectivement participé à l'APP expérimental en mars 2009 (cohorte 1) et au terme du même scénario utilisé dans le cadre régulier de l'APP en octobre 2009 (cohorte 2) dans le cadre de leur module endocrinologie. Le nombre d'items et de liens est significativement plus important pour les étudiants ayant participé à l'APP expérimental dès le début de la séance retour $(p<0,00001$ pour le nombre d'items et de liens) et on observe un gain nettement plus important pour ces mêmes étudiants ayant bénéficié des exercices de validation et délimitation du champ de leurs connaissances au cours de cette séance $(p=0,021$ pour le nombre d'items ; $p<0,001$ pour le nombre de liens). 
Tableau IV. Résultats du questionnaire relatif au fonctionnement du dispositif expérimental d'apprentissage par problèmes, administré à 20 étudiants participants répartis en trois groupes $\mathrm{A}(n=7), \mathrm{B}(n=7)$ et $\mathrm{C}(n=6)$.

--- : Pas du tout d'accord ; -- : Pas d'accord ; : :Plutôt pas d'accord ;+ : Plutôt d'accord ; ++ : D' accord ; +++ : Tout à fait d'accord \begin{tabular}{|l|c|c|c|c|c|c|c|}
\hline & \multicolumn{5}{|c|}{ Participants } \\
\hline Items & $p$ & --- & -- & - & + & ++ & +++ \\
\hline
\end{tabular}

Au terme de la phase de travail personnel

29. Mon travail était conforme à ce que je fais habituellement

\begin{tabular}{|c|c|c|c|c|c|c|c|}
\hline \multirow[t]{3}{*}{ 29. Mon travail était conforme à ce que je fais habituellement } & \multirow[t]{3}{*}{ NS } & & 14 & 14 & 29 & 29 & 14 \\
\hline & & & & & 14 & 29 & 57 \\
\hline & & & & & 83 & & 17 \\
\hline \multirow[t]{3}{*}{ 30. Je me suis plus investi que lors d'une séance APP habituelle } & \multirow[t]{3}{*}{ NS } & 14 & 29 & 43 & 14 & & \\
\hline & & & 14 & 43 & 43 & & \\
\hline & & & 33 & 50 & 17 & & \\
\hline \multirow[t]{3}{*}{ 31. Je me suis moins investi que lors d'une séance APP habituelle } & \multirow[t]{3}{*}{ NS } & 14 & 57 & 29 & & & \\
\hline & & 29 & 14 & 43 & 14 & & \\
\hline & & & 17 & $\mathbf{5 0}$ & 17 & 17 & \\
\hline
\end{tabular}

\section{Au cours de cette phase retour particulière}

\begin{tabular}{|c|c|c|c|c|c|c|}
\hline \multirow[t]{3}{*}{ 32. J'ai confirmé les éléments (items et liens) qui me paraissent connus } & \multirow[t]{3}{*}{$*$} & & & 14 & 43 & 43 \\
\hline & & & & & & 100 \\
\hline & & & & 17 & 67 & 17 \\
\hline \multirow{3}{*}{$\begin{array}{l}\text { 33. J'ai clarifié les éléments (items et liens) sur lesquels j'avais un doute ou une } \\
\text { hésitation }\end{array}$} & \multirow[t]{3}{*}{ NS } & & & 29 & 14 & 57 \\
\hline & & & & 14 & 14 & 72 \\
\hline & & & & 33 & 67 & \\
\hline \multirow{3}{*}{$\begin{array}{l}\text { 34. J'ai complété les parties qui me semblaient insuffisamment détaillées ou } \\
\text { développées }\end{array}$} & \multirow[t]{3}{*}{$*$} & & & 14 & 29 & 57 \\
\hline & & & & 14 & 29 & 57 \\
\hline & & 17 & & 33 & 50 & \\
\hline \multirow[t]{3}{*}{ 35. Le schéma coopératif m’a servi à identifier les limites de mes connaissances } & \multirow[t]{3}{*}{ NS } & 14 & & 29 & 43 & 14 \\
\hline & & & & 43 & 14 & 43 \\
\hline & & & 16 & 17 & 50 & 17 \\
\hline \multirow{3}{*}{$\begin{array}{l}\text { 36. Le schéma coopératif m'a servi à clarifier les éléments (items et liens) sur } \\
\text { lesquels j'avais un doute ou une hésitation }\end{array}$} & \multirow[t]{3}{*}{$*$} & & & 14 & 72 & 14 \\
\hline & & & & & 43 & 57 \\
\hline & & & & 33 & 67 & \\
\hline \multirow{3}{*}{$\begin{array}{l}\text { 37. Le schéma coopératif m’a servi à compléter les parties insuffisamment } \\
\text { détaillées ou développées }\end{array}$} & \multirow[t]{3}{*}{$\mathrm{NS}$} & & & & 67 & 33 \\
\hline & & & & 14 & 29 & 57 \\
\hline & & 17 & & 33 & 33 & 17 \\
\hline \multirow{3}{*}{$\begin{array}{l}\text { 38. Le schéma coopératif est un schéma de référence pour corriger mon schéma } \\
\text { individuel }\end{array}$} & \multirow[t]{3}{*}{$\mathrm{NS}$} & 17 & & 33 & 17 & 33 \\
\hline & & & & 14 & 14 & 72 \\
\hline & & 17 & & 33 & 33 & 17 \\
\hline \multirow[t]{3}{*}{ 39. Le schéma coopératif permet de valider mon raisonnement } & \multirow[t]{3}{*}{ NS } & & & 28 & 43 & 29 \\
\hline & & & & & 29 & 71 \\
\hline & & & & 50 & 33 & 17 \\
\hline \multirow{3}{*}{$\begin{array}{l}\text { 40. Cerner ce que je sais et les limites de mes connaissances m'a permis une } \\
\text { meilleure intégration de mes connaissances }\end{array}$} & \multirow[t]{3}{*}{ NS } & & & 29 & 71 & \\
\hline & & & & 14 & 57 & 29 \\
\hline & & & & 50 & 50 & \\
\hline \multirow{3}{*}{$\begin{array}{l}\text { 41. Cerner ce que je sais et les limites de mes connaissances m'a forcé à un } \\
\text { apprentissage plus en profondeur }\end{array}$} & \multirow[t]{3}{*}{ NS } & & & 17 & $\mathbf{5 0}$ & 33 \\
\hline & & & 14 & 57 & & 29 \\
\hline & & & & 50 & 17 & 33 \\
\hline \multirow{3}{*}{$\begin{array}{l}\text { 42. Cerner ce que je sais et les limites de mes connaissances m'a permis d'identifier } \\
\text { les limites de mon raisonnement }\end{array}$} & \multirow[t]{3}{*}{ NS } & & & 17 & 83 & \\
\hline & & & & 29 & 43 & 28 \\
\hline & & & & 67 & 17 & 16 \\
\hline
\end{tabular}

$* p<0,05$, différence significative entre les trois groupes.

NS : non significat. 


\begin{tabular}{|c|c|c|c|c|c|c|c|c|c|c|c|c|c|c|c|c|c|c|c|}
\hline & & $\begin{array}{l}+ \\
+ \\
+ \\
+\end{array}$ & $r$ & $\simeq$ & 으 & \pm & $n$ & $r$ & & & $N$ & $\sim$ & in & $\sim$ & in & & in & & $n$ \\
\hline & & + & $\exists$ & in & i & in & $r$ & $m$ & & $\infty$ & $\ddot{m}$ & $\bar{n}$ & $\vec{\sim}$ & $\bar{\sim}$ & \pm & đ & $\stackrel{\sim}{2}$ & ৯े & $\stackrel{i}{\sim}$ \\
\hline - & : & + & F & $\stackrel{\infty}{\sim}$ & $\stackrel{\infty}{\sim}$ & iి & $\widehat{\imath}$ & F & & $\mathcal{F}$ & \& & $\widehat{\lambda}$ & $\vec{\sim}$ & $\infty$ & $\vec{\sim}$ & $F$ & तิ & $m$ & $F$ \\
\hline 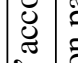 & 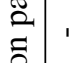 & & $N$ & in & లి & in & in & $\simeq$ & & $\bar{\sim}$ & $a$ & $\Xi$ & సิ & 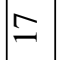 & $\ddot{m}$ & $\vec{\sim}$ & 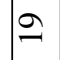 & $\stackrel{\sim}{\sim}$ & 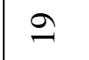 \\
\hline : & & ; & $n$ & $\mathrm{~N}$ & N & $N$ & $\simeq$ & 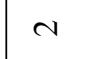 & & $\curvearrowright$ & $\simeq$ & $r$ & $\equiv$ & \pm & $\beth$ & $a$ & \pm & $n$ & $\sim$ \\
\hline$\stackrel{\sim}{\Xi}$ & & : & & & & & & & & $\sim$ & $N$ & N & $r$ & $a$ & 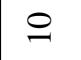 & $n$ & $r$ & $r$ & $r$ \\
\hline$\ddot{+}$ & & $\begin{array}{l}+ \\
+ \\
+\end{array}$ & 으 & 18 & & $n$ & & ㄴ. & & $\cong$ & $ㅇ$ & 으 & 으 & 으 & $n$ & $n$ & in & in & 으 \\
\hline $\begin{array}{l}+ \\
\therefore\end{array}$ & & + & 18 & 18 & 우 & $\ddot{n}$ & & 8 & & f & 6 & 17 & $\cong$ & $\approx$ & & i & 网 & $\tilde{m}$ & $\tilde{n}$ \\
\hline $\mid \begin{array}{l}0 \\
0 \\
0\end{array}$ & $\begin{array}{l}\overrightarrow{\tilde{z}} \\
\underline{2}\end{array}+$ & + & 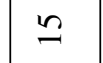 & 은 & 18 & i & $\ddot{v}$ & $=$ & & ஓ & $\mathscr{\imath}$ & $\cong$ & $\mathbb{1}$ & in & 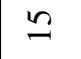 & 8 & in & in & fo \\
\hline 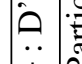 & : & ' & 으 & & 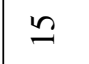 & & 8 & & & 0 & & & $\stackrel{\sim}{~}$ & $n$ & in & in & $\cong$ & in & in \\
\hline $\begin{array}{l}+ \\
\therefore\end{array}$ & & ; & & & & & 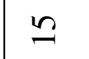 & & & & & & $\cong$ & $n$ & iి & & & & $n$ \\
\hline$\ddot{U}$ & & 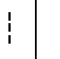 & & & & & & & & in & & & $\cong$ & in & in & & & & \\
\hline$\overline{0}$ & & 2 & $\tilde{z}$ & $*$ & $\tilde{z}$ & $\tilde{z}$ & $\tilde{z}$ & * & & $*$ & & * & $\tilde{z}$ & $\tilde{z}$ & $\tilde{z}$ & & $\tilde{z}$ & 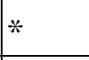 & $\tilde{z}$ \\
\hline 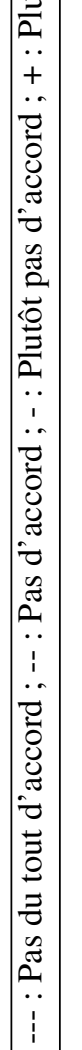 & & $\stackrel{\mathscr{E}}{\mathscr{E}}$ & 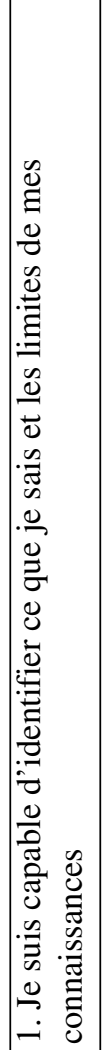 & 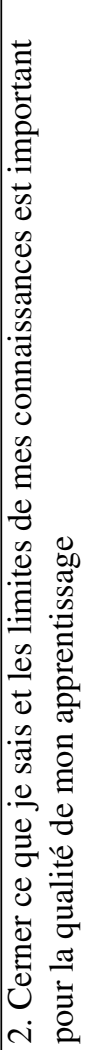 & 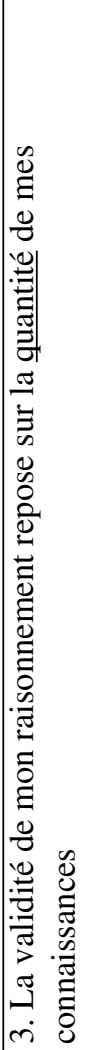 & 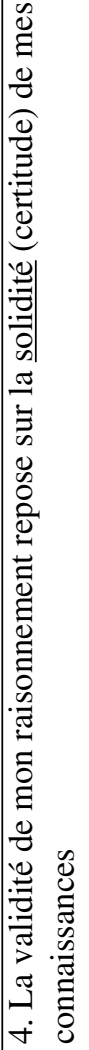 & 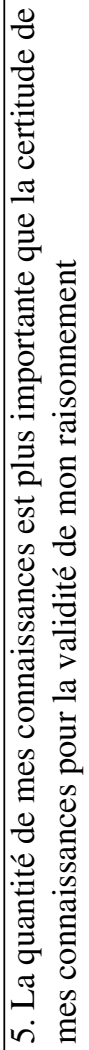 & 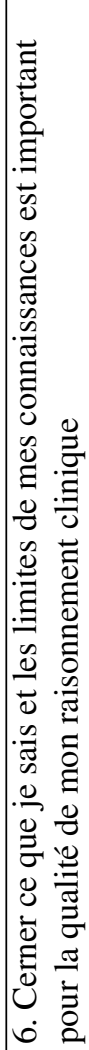 & 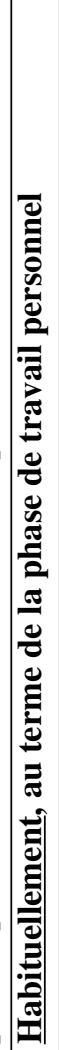 & 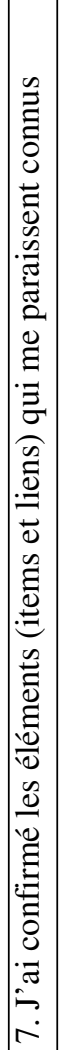 & 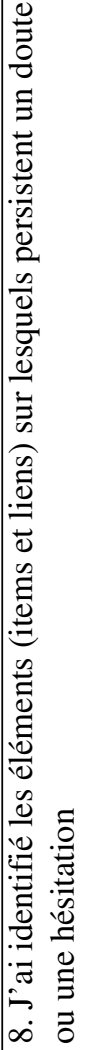 & 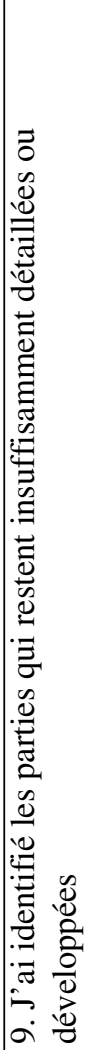 & 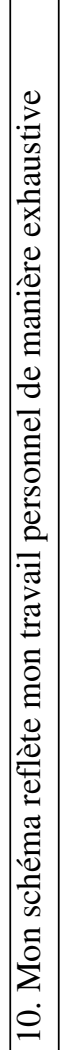 & 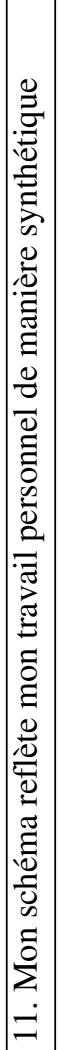 & 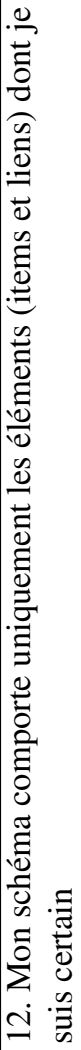 & 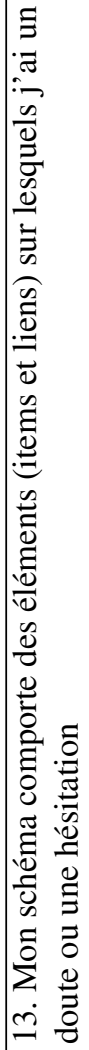 & 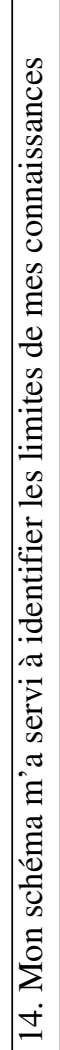 & 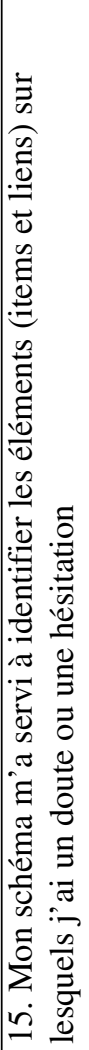 & 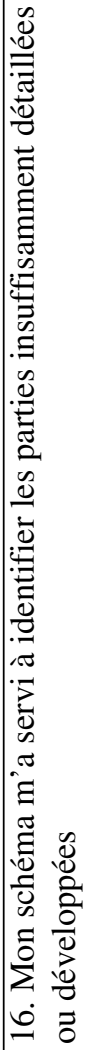 \\
\hline
\end{tabular}




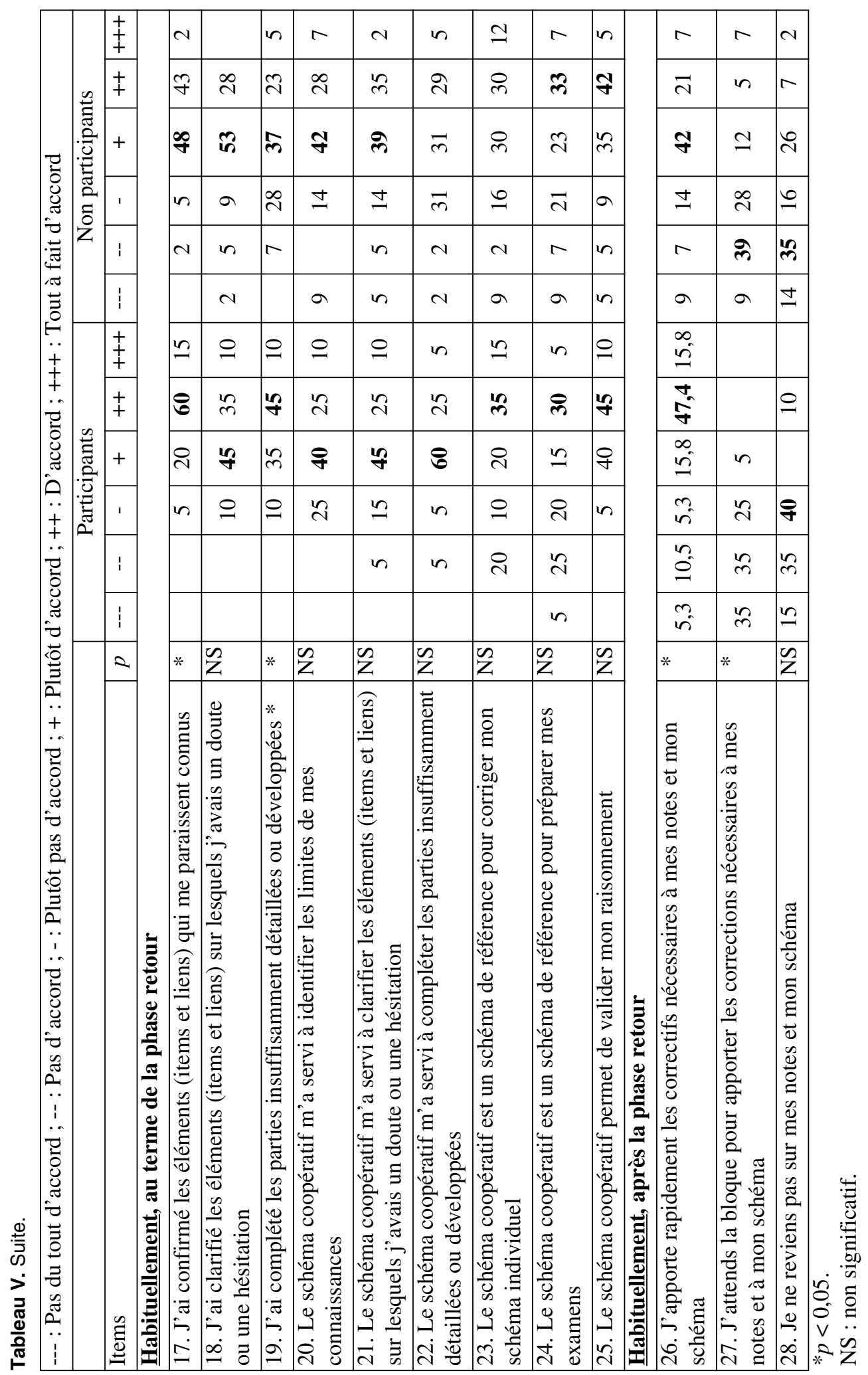




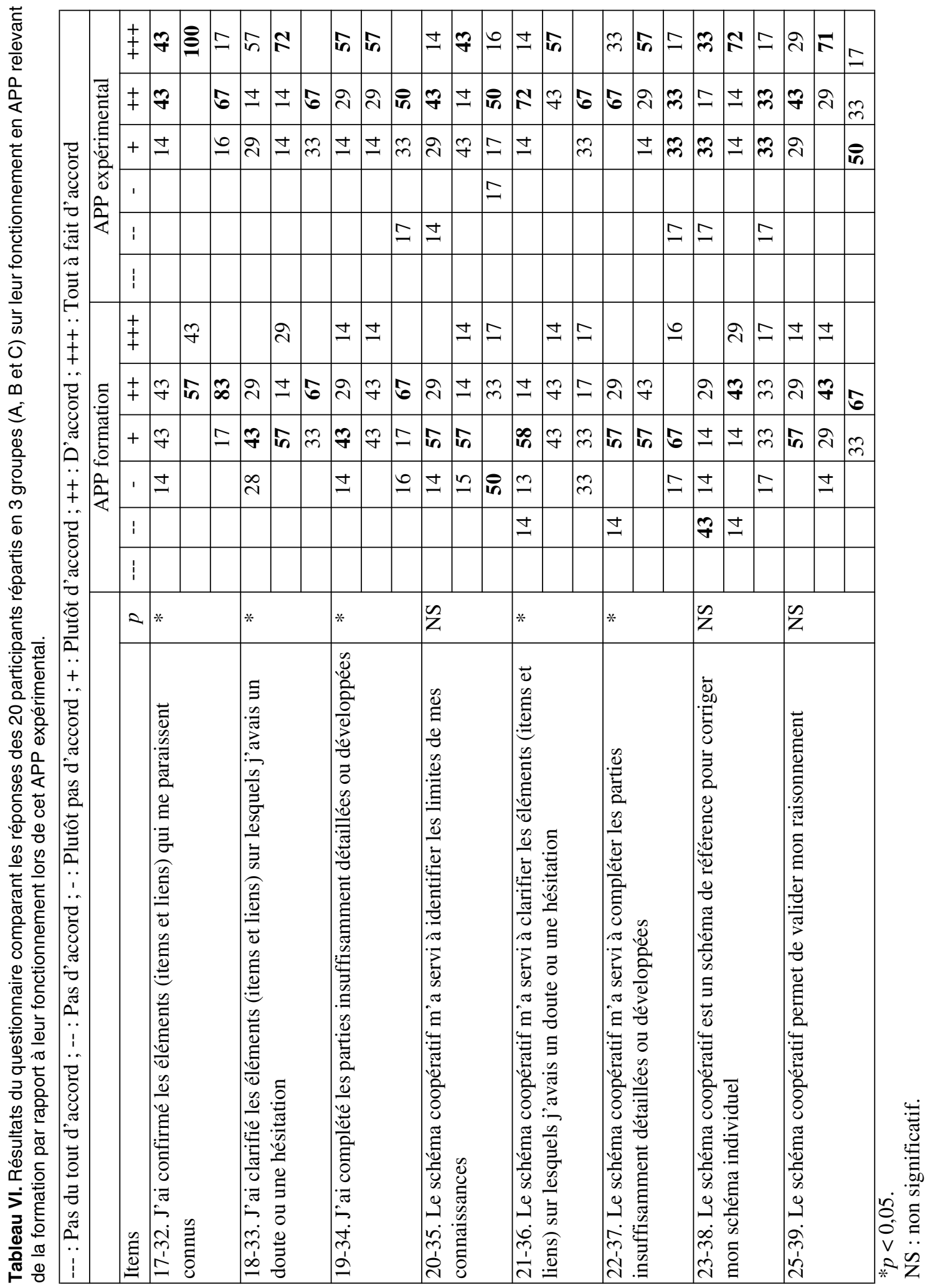



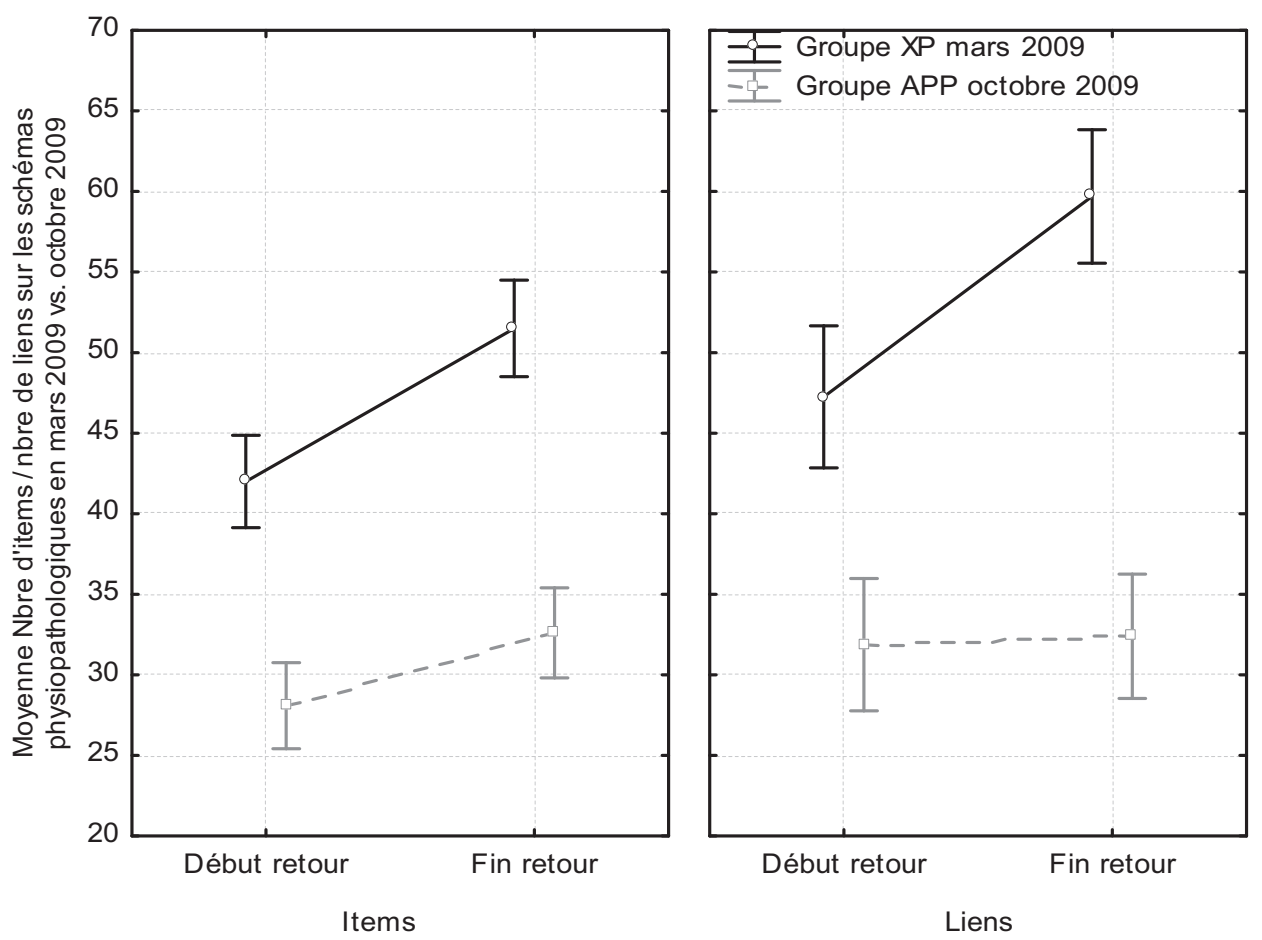

Fig. 1. Moyenne ( \pm Ecart type) du nombre d'items (à gauche) et de liens (à droite) figurés dans les schémas individuels du groupe ayant participé à l'étude 1 en mars 2009 (XP) et de la cohorte suivante ayant suivi la séance d'apprentissage par problèmes (APP) en octobre 2009 en début et en fin de séance « retour ».

\section{Discussion}

L'objectif général de cette étude était de documenter les effets d'une démarche explicite d'auto-validation des connaissances, proposée à des étudiants dans le cadre d'un séminaire recourant aux principes et au format de l'APP.

Apprécié au regard du nombre d'items et de liens figurés dans les schémas physiopathologiques élaborés par les étudiants des différents groupes aux différents moments de la séance retour, aucun impact n'est observé avant la mise en commun coopérative. En revanche, les schémas coopératifs construits par les groupes et les schémas individuels récoltés après la mise en commun comprennent plus d'items et de liens dans le groupe A que dans les autres groupes. Nous pourrions faire l'hypothèse que ce groupe, ne sachant toujours pas à ce stade du séminaire l'objectif de la recherche mais étant par ailleurs informé du caractère expérimental de celui-ci, était plus attentif à un schéma complet afin de pouvoir répondre à d'éventuelles questions à la suite de la mise en commun. La façon dont la séance a été menée par le tuteur pourrait également avoir pu induire une utilisation différente des informations de la vignette dans les schémas.

Les exercices de délimitation du champ des connaissances ne semblent pas avoir modifié de manière significative les schémas individuels des différents groupes. En effet, nous ne pouvons pas attribuer aux exercices de délimitation l'évolution des schémas individuels entre le début et la fin de la séance « retour » car aucune différence significative n'est observée entre le groupe A et les deux autres groupes à ces différentes étapes.

Quelques différences sont observées relativement à la façon dont les différents groupes ont marqué leurs schémas durant les exercices de délimitation. Les étudiants du groupe $\mathrm{C}$ ont marqué davantage d'éléments douteux que ceux du groupe 
B. Nous pourrions faire l'hypothèse que ces étudiants, ayant été prévenus qu'ils auraient à mettre en évidence sur leurs schémas les éléments dont ils étaient sûrs et ceux dont ils doutaient, ont été incités à noter plus d'éléments même s'ils en doutaient. Par ailleurs, lors du deuxième exercice de validation, les étudiants du groupe A ont marqué beaucoup plus d'éléments manquants ou incomplets que les groupes $\mathrm{B}$ et $\mathrm{C}$. Ces derniers avaient réalisé avant le schéma coopératif l'exercice 1 au cours duquel ils avaient dû marquer les éléments insuffisamment détaillés ou développés. Il faut d'ailleurs remarquer que l'observation est similaire si la consigne « validation des éléments du schéma » est donnée avant ou après la phase de travail personnel. Logiquement, ces deux groupes avaient également ajouté moins d'éléments lors de la phase de mise en commun. Lorsque nous comparons l'ensemble des éléments marqués au cours des deux exercices par les trois groupes, nous n'observons plus de différence entre les groupes. Si le résultat final est donc comparable, il résulte davantage d'un processus individuel chez les étudiants invités à valider leur schéma avant la mise en commun. On peut s'interroger sur l'impact de cette différence à plus long terme.

Les résultats du questionnaire indiquent, chez les étudiants volontaires ayant participé à l'APP expérimental, un investissement plus important dans leur apprentissage et une plus grande conscience de l'importance de la validation des connaissances dans le cadre de cet apprentissage et du développement du raisonnement clinique. Ils indiquent également que les exercices proposés leur permettent de mettre davantage en évidence l'évolution de leur apprentissage. Bien qu'il ait été créé en cohérence avec les consignes et en respectant le canevas et les objectifs des séances, et bien qu'il ait été préalablement approuvé par un groupe d'enseignants, ce qui permet de lui attribuer une certaine validité, les qualités psychométriques du questionnaire n'ont pas été formellement vérifiées, ce qui constitue une limite théorique à l'analyse statistique des réponses recueillies. Toutefois, les commentaires recueillis lors du débriefing viennent corroborer les interprétations effectuées à partir des réponses au questionnaire. Par ailleurs, ce questionnaire étant rempli après l'APP expérimental, les réponses des étudiants participants peuvent avoir été influencées par la procédure expérimentale même si les questions sont explicitement posées à propos des autres séminaires.

Ainsi, la mise en commun autour du schéma coopératif semble avoir un impact positif pour la validation/correction du schéma et des apprentissages par les étudiants. Toutefois, ce processus de validation/ correction concrétisé dans le schéma peut avoir impliqué l'étudiant plus ou moins activement et on ne peut exclure que certains retranscrivent passivement la production des autres étudiants. L'impact direct des exercices de délimitation du champ des connaissances sur la production finale ne peut donc pas être identifié formellement. Par contre, l'analyse de schémas produits avant la mise en commun de la séance « retour » permet de documenter les effets de la formulation aux étudiants d'une consigne explicite de validation de leur propre production. Il est raisonnable de faire l'hypothèse que la formulation itérative d'une telle consigne inciterait les étudiants à travailler de manière personnelle leur propre base de connaissances, en favorisant le processus individuel plutôt que le seul recours au travail collectif.

La nature du processus de délimitation du champ des connaissances mérite aussi d'être discutée: il pourrait s'agir d'un processus complexe déjà maîtrisé chez l'étudiant qui l'applique de manière implicite dans le cadre de ses APP, quelle que soit la consigne ou, au contraire, d'un processus nouveau, suscité et favorisé par une consigne explicite de validation conduisant à la délimitation du champ des connaissances.

L'utilisation de la vignette créée pour cette séance d'APP expérimental, dans le cadre de la formation de la cohorte suivante, met en évidence des différences dans l'utilisation des schémas physiopathologiques d'un groupe à l'autre.

La différence de niveau de base du schéma de début de séance «retour» entre les deux cohortes d'étudiants peut s'expliquer, du moins en partie, par les périodes de séance APP différentes. Effectivement, le groupe expérimental de la première cohorte avait déjà suivi l'ensemble du module endocrinométabolisme le quadrimestre précédent l'expérimentation et avait également déjà subi l'évaluation de cette matière. Par contre, la cohorte 2 était en cours 
de module et avait donc investi moins de travail dans la matière dont elle n'avait pas encore une vision d'ensemble à ce stade. Une différence de connaissances de base en endocrinologie mais aussi d'expérience en termes de nombres de séminaires APP suivis peut contribuer à expliquer cette différence. Un facteur supplémentaire peut être lié à la motivation, les étudiants de l'APP expérimental sachant qu'ils participaient à un séminaire APP dans lequel une procédure nouvelle serait introduite, alors que les étudiants de l'APP comparatif avaient simplement donné leur aval pour la communication et l'utilisation de leurs schémas.

Le gain en cours de séance, qui est plus important pour le groupe expérimental de la cohorte 1, peut s'expliquer par différents facteurs. Tout d'abord, comme nous avons pu le voir dans la première partie de l'étude, les participants à l'APP expérimental étaient des étudiants déjà plus impliqués dans la méthode APP que les étudiants non participants. Ensuite, la consigne de l'APP expérimental incitait à corriger le schéma et à se poser des (bonnes) questions avant la mise en commun plutôt qu'à simplement recopier le schéma du groupe. Enfin, les étudiants ayant déjà étudié l'ensemble de la matière ont créé un réseau de connaissance plus complet mais aussi plus structuré, qui permet une meilleure appréhension du problème et une confrontation facilitée au réseau de connaissance créé.

\section{Conclusion}

Cette étude avait pour objectif d'évaluer l'effet d'un travail d'auto-validation des connaissances de l'étudiant sur son apprentissage et sur la validation de son raisonnement dans le cadre d'un dispositif de type APP. Dans la mesure où les séances d'APP se déroulent en groupe, il n'est pas toujours évident pour les étudiants, en particulier les moins investis, de prendre le temps de s'interroger de façon individuelle sur leurs propres limites de compréhension des concepts clés liés au problème. Souvent, il n'y a pas d'incitation systématique de la part de l'enseignant à faire entrer l'étudiant dans cette démarche. Nos résultats suggèrent qu'un exercice de validation et de délimi- tation du champ des connaissances avant la mise en commun collective force l'étudiant à évaluer sa propre production et à questionner ses propres connaissances. Un tel exercice pourrait s'avérer particulièrement utile pour les étudiants qui sont moins actifs et participatifs lors des séances d'APP. Notre étude conduit également à recommander d'intégrer aux dispositifs pédagogiques de type APP davantage de moments de réflexion individuelle et de rendre explicite le travail de validation des connaissances sous-jacentes au raisonnement d'analyse des situations cliniques, afin de renforcer la structuration des connaissances et ainsi développer les capacités de raisonnement.

\section{Contributions}

Tous les auteurs ont contribué de façon équivalente à la conception de la recherche, au recueil et à l'interprétation des données ainsi qu'à l'écriture du manuscrit.

\section{Déclaration d'intérêts}

Aucun auteur ne déclare de conflit d'intérêt en lien avec le contenu de cet article.

\section{Approbation éthique}

Non sollicitée

\section{Références}

1. Boniver J. Les études de médecine à l'Université de Liège : le renouveau pédagogique de la Faculté de Médecine. Rev Med Liege 2004;59:717-30.

2. van Gessel E, Nendaz MR, Vermeulen B, Junod A, Vu NV. Development of clinical reasoning from the basic sciences to the clerkships: a longitudinal assessment of medical students' needs and self-perception after 
a transitional learning unit. Med Educ 2003;37:96674.

3. Schmidt HG. Problem-based learning: rationale and description. Med Educ 1983;17:11-6.

4. Norman GR, Schmidt HG. The psychological basis of problem-based learning: a review of the evidence. Acad Med 1992;67:557-65.

5. Regehr G, Norman GR. Issues in cognitive psychology: implications for professional education. Acad Med 1996;71:988-1001.

6. Davis M, Harden R. AMEE Medical Education Guide No. 15: Problem-based learning: a practical guide, Med Teach 1999;21:130-40.

7. Newble DI, Clarke RM. The approaches to learning of students in a traditional and in an innovative problem-based medical school. Med Educ 1986;20: 267-73.

8. Barrows H, Pickell G. Developing Clinical Problem Solving Skills: A Guide to More Effective Diagnosis and Treatment. New York: Norton, 1991.

9. Barrows H. The scope of clinical education. J Med Educ 1986;61:23-33.

10. Schwartz RW, Burgett JE, Blue AV, Donnelly MB, Sloan DA. Problem-Based Learning and Performance-Based Testing: Effective Alternatives for Undergraduate Surgical Education and Assessment of Student Performance. MedTeach 1997;19:19-23.

11. Friedman CP, deBliek R, Greer D, Mennin S, Norman $\mathrm{G}$, Sheps $\mathrm{C}$, et al. Charting the winds of change: evaluating innovative medical curricula. Acad Med 1990;65:8-14.

12. Haak DC, KillRisLambers J, Pitre E, Freeman S. Increased Structure and Active Learning Reduce the Achievement Gap in Introductory Biology. Science 2011;332:1213-16.

13. Yeung E, Au-Yeung S, Chiu T, Mok N, Lai P. Application of Problem Based Learning Strategies to Enhance Clinical Reasoning and Self-Directed Learning Skills in a University Physiotherapy Program. In: Marsh J (Ed.) Implementing Problem Based Learning Project: Proceedings of the First Asia Pacific Conference on Problem Based Learning.
Hong Kong: The University Grants Committee of Hong Kong, Teaching Development Project, 1999: 95-110.

14. Collard A, Gelaes S, Vanbelle S, Bredart S, Defraigne JO, Boniver J, Bourguignon JP. Reasoning versus knowledge retention and ascertainment throughout a problem-based learning curriculum. Med Educ 2009;43:854-65.

15. Jones MA. Clinical resoning in manual therapy. Phys Ther 1992;72:875-84.

16. Langendyk V. Not knowing that they do not know: self assessment accuracy of third-year medical students. Med Educ 2006;40:173-9.

17. Elstein AS, Shulman IS, Sprafka SS. Medical Problem Solving: An Analysis of Clinical Reasoning. Cambridge (MA): Harvard UniversityPress, 1978.

18. Elstein AS, Shulman LS, Sprafka SA. Medical problem solving: a ten-year retrospective. Eval Health Prof 1990;13:5-36.

19. Gale J. Some cognitive components of the diagnostic thinking process. Br J Educ Psychol. 1982;52:64-76.

20. Nickerson RS, Perkind, DN, Smith EE. The Teaching of Thinking. Hillsdale (NJ): Lawrence Erlbaum Assosiates INC, 1985.

21. Mamede S, Schmidt HG, Rikers R. Diagnostic errors and reflective practice in medicine. J Eval Clin Pract 2007;13:138-45.

22. Collard A, Mélot F, Bourguignon JP. Longitudinal assessment of progress in reasoning capacity and relation with self-estimation of knowledge base. Assess Eval High Educ 2014, in press.

23. Chang RW, Bordage G, Connell KJ. The importance of early problem representation during case presentations. Acad Med 1998;73:S109-11.

24. Nendaz M, Charlin B, Leblanc V, Bordage G. Le raisonnement clinique: données issues de la recherche et implications pour l'enseignement. Pédagogie Médicale 2005;6:235-54.

Correspondance et offprints: Anne Collard, Institut de formation et de recherche en enseignement supérieur (IFRES), B63b, Traverse des architectes, 4000 Liège, Belgique; Mailto : acollard@ulg.ac.be 